\title{
Adherence to the Core Elements of Outpatient Antibiotic Stewardship: A Cross-Sectional Survey in the Tertiary Care Hospitals of Punjab, Pakistan
}

Maryam Raheem (ID

Sidra Anwaar (D)

Zanish Aziz

Sarwat Ali Raja

Nasira Saif-ur-Rehman

Naeem Mubarak (D)

Lahore Pharmacy College, Lahore Medical \& Dental College, University of Health Sciences, Lahore, Punjab, Pakistan

Correspondence: Sarwat Ali Raja; Naeem Mubarak

Email sarwat.ali@Imdc.edu.pk; naeem. mubarak@Imdc.edu.pk
This article was published in the following Dove Press journal: Infection and Drug Resistance

Background: There is a growing global interest in hospital-based antibiotic stewardship programs (ASPs). Centers for Disease Control and Prevention (CDC) recommends clinicians and facilities in outpatient department (OPD) to adhere to a set of stewardship activities called the Core Elements of Outpatient Antibiotic Stewardship (CEOAS). CEOAS includes 4 core elements for OPD facilities and clinicians each, ie, commitment, action, tracking and reporting, and education and expertise.

Aim: The aim of this study was to evaluate the adherence of OPDs in tertiary care hospitals to CEOAS.

Design and Setting: A cross-sectional study in the hospitals in Punjab, Pakistan.

Methods: Study was reported as per STROBE guidelines. Data were collected from hospitals based on purposive sampling on the CEOAS framwork. On a summative scale, positive response to each core element worthed a score and higher the score better the adherence. Descriptive statistics was used for categorical variables while independent $t$-test computed group differences.

Results: Fifty-three tertiary care hospitals ( $n=22$ public, $n=31$ private) participated (response rate $=86.9 \%$ ). No hospital reported "perfect" adherence. Overall, facilities and clinicians in OPDs were moderately adherent. Subgroup analysis indicated that hospitals in public and private were poorly (4.9) and moderately (6.0) adherent to CEOAS respectively, however, private clinicians scored significantly higher in action, and tracking and reporting. Tracking and reporting of antibiotics and education of patients and clinicians emerged as top deficiency areas in facilities and clinicians respectively.

Conclusion: Significant gaps exist in the adherence to CEOAS. The deficiency areas highlighted in the study should be given priority in future policy shift.

Keywords: antibiotic stewardship, antimicrobial stewardship, hospitals, Pakistan, outpatient department; OPD, core elements, CDC

\section{Background}

Antibiotic Resistance and Antibiotics Stewardship Programs In this continuously changing world, bacteria also have evolved to develop resistance against the antibiotics — once considered a miracle cure. This means antibiotics would no longer be effective against resistant bacteria - whom World Health Organization — termed as "nightmare bacteria." These nightmare bacteria make even common infections complicated, limit the treatment options, increase mortality, morbidity and hospital stays and consume more health care resources. ${ }^{1}$ 
The interconnectedness of the modern world has proven to be a boon for the phenomenon of bacterial resistance that has now spread across the continents as a "catastrophic threat" to people, health care system and economy in every country in the world. ${ }^{2}$

Coordinated actions, strategies and policies are part of global agenda to contain the threat of antimicrobial resistance. Two contributing - yet modifiable - factors in the development of resistance in bacteria include inappropriate prescribing and inappropriate use of antibiotics. ${ }^{3}$ This entails implementation of stringent policies and efforts that could modify the prescribing habits of clinicians to an evidence base practice to ensure antibiotics are only prescribed when needed. Antimicrobial or antibiotic stewardship programs (ASPs) are:

coordinated interventions designed to improve and measure the appropriate use of [antibiotic] agents by promoting the selection of the optimal [antibiotic] drug regimen including dosing, duration of therapy, and route of administration. ${ }^{4}$

Usually based on a local or international policy, guidelines or recommendation, ASPs are planned, coordinated and implemented by a health care system or part of the system such as an outpatient department (OPD) of a hospital. $^{5}$ All health care settings (where antibiotics are prescribed) require ASPs, however, in outpatient setting this requirement becomes critical because $80-90 \%$ antibiotics are used in outpatient setting in developed countries. ${ }^{6,7}$

United States, Centers for Disease Control and Prevention (CDC) has defined core components of a universally acceptable ASP applicable specifically to outpatient setting known as the Core Elements of Outpatient Antibiotic Stewardship (CEOAS). CDC recommends OPD clinicians and facilities to adhere to the recommended CEOAS (Appendix A1, Table A1 and A2). ${ }^{8}$ WHO also reinforces the importance of ASPs to be implemented in hospitals and consequently, in many countries recent initiatives require hospitals - no matter whether in a resource limited or resource rich setting to implement some form of ASPs. However, in Lower- to Middle-Income Countries (LMIC) the concept of antibiotic stewardship has received recent attention. ${ }^{9,10}$

\section{Situation in Pakistan}

Pakistan - the 5th most populous country in the world belongs to world bank strata of LMIC. More than $65 \%$ population of Pakistan $(110,012,442)$ lives in the province of Punjab, where Lahore is the provincial capital. Administratively, the province of Punjab has 9 divisions with roughly 147 hospitals (49 public hospitals). A large fraction seeks health care in the public sector hospitals where an estimated 70,000 patients attend OPDs per day. Similarly, in Lahore alone, roughly 20,000 people visit OPDs per day. ${ }^{11,12}$

Recent point prevalence surveys on antibiotic use in hospitals of Punjab have raised many red flags on the high rates of antibiotic prescribing (more than 3 drugs per patient) and consumption. ${ }^{13,14}$ Furthermore, roughly $70 \%$ patients receive antibiotics, and development of resistance in various strains of bacteria have been reported extensively. ${ }^{15,16}$ However, the fact is, situation is underreported and health authorities in Pakistan are concerned on the inexorable rise of the antibiotics' consumption and resultant rife in the antibiotic resistance over the last decade. Pakistan's national action plan aims to optimize the use of antibiotics through stewardship activities with a goal to sustain the effectiveness of current plethora of antibiotics as long as possible. Hence, health authorities urge hospitals nationwide to develop and implement ASPs. ${ }^{15}$ However, there is a paucity of research on evaluations of the extent to which OPDs in hospitals adhere to ASPs. In context of CDC framework CEOAS the research questions posit:

1- What percentage of tertiary care private and public hospitals in Punjab are in the category of poor, moderate, good, or perfect as defined based on summative scale?

1. 2- Is there any difference between the mean scores of private and public hospitals for facilities and clinicians' indicators?

2. 3- Based on findings, what are the priority areas for future policy and practice.

\section{Research Objectives}

This study aims to evaluate adherence of OPDs in tertiary care hospitals to CDC proffered CEOAS, to inform the relevant stakeholders about the current situation and future implication for any policy shift regarding hospital-based ASPs.

\section{Methods}

\section{Design, Setting and Survey Instrument}

It was a cross-sectional study to collect institutional data of the hospitals in Punjab, Pakistan, based on purposive sampling. Contact details of the hospitals were obtained 
from Director General of Health Services Office, Punjab. CDC recommended CEOAS framework was used as a survey instrument. A core element was defined as a broad category of action or strategy (e.g., commitment or education) for stewardship. The reasons we preferred to deploy CDC recommended CEOAS as a survey instrument to define extent of adherence, despite availability of various other check lists, include:

1. It was specifically designed for OPD facilities and clinicians.

2. Contents of the survey were generic and applicable to any outpatient setting whether in resource rich or resource poor setting.

3. It was a validated instrument which highlights ASPs in two different ways, from the angle of clinicians and from the aspect of physical facilities offered by a given hospital.

The survey instrument had three parts:

1. The demographic details of the hospital (name, ownership (public/private), name of the division in Punjab, and sign and stamp of the hospital representative filling the survey)

2. Core elements for outpatient Facilities, referred as facilities indicators with a total possible score 12 .

3. Core elements for outpatient Clinicians, referred as clinicians' indicators with a total possible score 18 .

Both, facilities, and clinicians' indicators were defined by four main core elements each and include: Commitment, Action for policy and practice, Tracking and reporting, and Education and expertise (details, Appendix A1). A unique core element was covered by a 3 to 4 statements (items) in the form a check list, where each statement carried a weight of 1 or 0 score (numerical variable, interval) if answered "YES" or "NO" respectively. Based on a summative scale, if choose to answer YES for all the statements of the survey, a hospital could achieve a maximum score of 18 and 12 for facilities and clinicians' indicators, respectively. The mean scores of hospitals would be related to adherence where higher the score, better would be adherence to CEOAS. To evaluate the extent of the adherence of OPDs to CEOAS for facilities and clinicians, the total score (mean) obtained by the hospitals were assigned a category to define level of adherence, for instance for facilities, total score range of $0-4.9,5.0-9.9$, 10.0-15.9, and 16.0-18.0 were denoted "poor," "moderate," "good," and "perfect," respectively. Similarly, for clinicians' indicators, total score range of 0-3.9, 4.0-6.9, 7.0-9.9, and 10.0-12.0 were assigned "poor," "moderate," "good," and "perfect," respectively. Study was reported in accordance to STROBE guidelines (Table A3-Appendix A1)

\section{Data Collection}

Prior to data collection, three Doctor of Pharmacy, final year students were trained to collect data. The trained students took appointments for a personal meeting from the administration of the hospitals willing to participate in the survey. We aimed to reach tertiary care hospitals located in all the 9 geographical and administrative divisions of the Punjab. Data were collected in person on the validated CDC, CEOAS framwork checklist, the survey instrument, from 17th December 2019, to 15th January 2020. The data were collected in person, self-administered, from any of the following representative of the hospital who signed and stamped the survey after completion: Medical Superintendent (MS) or Deputy or Assistant Medical Superintendent (DMS/AMS) or Manager/Head of pharmacy or Chief Pharmacist (CP) of the hospitals. Data collection team members remained present throughout the meeting to explain or clarify any statement in proforma. In person, selfadministration of the survey offered valuable advantage of error free recording of the responses. Only those proformas were added in the final analysis which were signed and stamped by any of the earlier mentioned dignitaries.

\section{Inclusion and Exclusion Criteria}

All tertiary care hospitals located in Punjab were eligible to participate in the survey. In context of this study, a tertiary care hospital was defined as "any hospital enlisted by the Punjab government as an access point for tertiary care OR any hospital with distinct specialities and facilities, such as ICU, CCU, Dialysis, ventilators, in house lab testing and pharmacy services, etc., besides, general specialties like surgery, medicine, orthopedics, pediatrics, and gynae."

Tertiary care hospitals $<30$ beds, secondary care hospitals and primary care clinics were excluded.

\section{Data Management and Statistical Analysis}

Data were managed and analyzed in Statistical Package Social Sciences (version 22 IBM, California, USA). Descriptive statistics were used to evaluate the extent of adherence and $t$-tests were performed to analyze the mean difference between private and public hospitals for each of the core element mentioned earlier with a $p$ value $<0.05$ considered significant. 


\section{Ethics}

The Research Ethics Committee, Lahore Pharmacy College, Lahore Medical \& Dental College granted ethical approval of the study (ref\# ETH/LPC/10/10/19). After detailing about the aims and objectives of the research, informed consent was taken from the representative of all participating hospitals. To ensure the anonymity, hospitals were assigned individual identification numbers and data were stored in a password protected computer.

\section{Results}

A total of $\mathrm{N}=53$ tertiary care hospitals $(\mathrm{n}=22$ public, $\mathrm{n}=31$ private) participated in the survey. The response rate was $86.9 \%$ (6 private and 2 public hospitals declined due to lack of willingness to share the data). Survey was mainly responded by MS (56.6\%), followed by DMS/AMS (24.5\%) and CP (18.9\%). Participating hospitals were diversely located in 8 out of 9 administrative divisions of the province of Punjab namely: Lahore, Faisalabad, Bahawalpur, Multan, Rawalpindi, Gujranwala, Sargodha, and Sahiwal besides Islamabad Capital Territory (the federal capital). As the original CDC proforma separately covers clinicians and facilities indicators, hence for the sake of clarity and to identify specific areas of deficiency, this study also reported the results of extent of adherence separately for clinicians and facilities of the included hospitals. Overall, both OPD facilities and clinicians reported "Moderate" adherence to CEOAS.
Not a single hospital, whether in private or public sector was able to demonstrate "perfect" adherence, neither for the clinician's indicators nor for the facilities indicators. The response on the individual items in the survey can be found in the Table A1 for facilities and Table A2 for clinicians in Appendix A1.

\section{OPD Facilities Indicators}

The majority of the facilities in public (59.1\%) and private hospitals $(64.5 \%)$ were in the "Moderate" category as depicted in Figure 1.

Overall mean score (5.58) implied that facilities in all hospitals were in the category of "Moderate." While in subgroup analysis, mean scores indicated hospitals in public and private were poorly (4.9) and moderately (6.0) adherent to CEOAS respectively. However, the difference in mean score between the two groups was not statistically significant $(p=0.37)$. Furthermore, mean score for all the individual core elements (commitment, action, tracking and reporting, and, education and expertise) was higher in private as compared to public hospitals, nevertheless, only tracking and reporting showed significant difference $(\mathrm{p}=0.05)$ as detailed in Table 1 .

\section{OPD Clinicians Indicators}

The majority of the OPD clinicians in public (45.5\%) and private hospitals $(51.6 \%)$ hospital were in the "Good" category as depicted in Figure 2.

Based on overall mean score (6.43), clinicians in all hospitals were moderately adherent to CEOAS. In

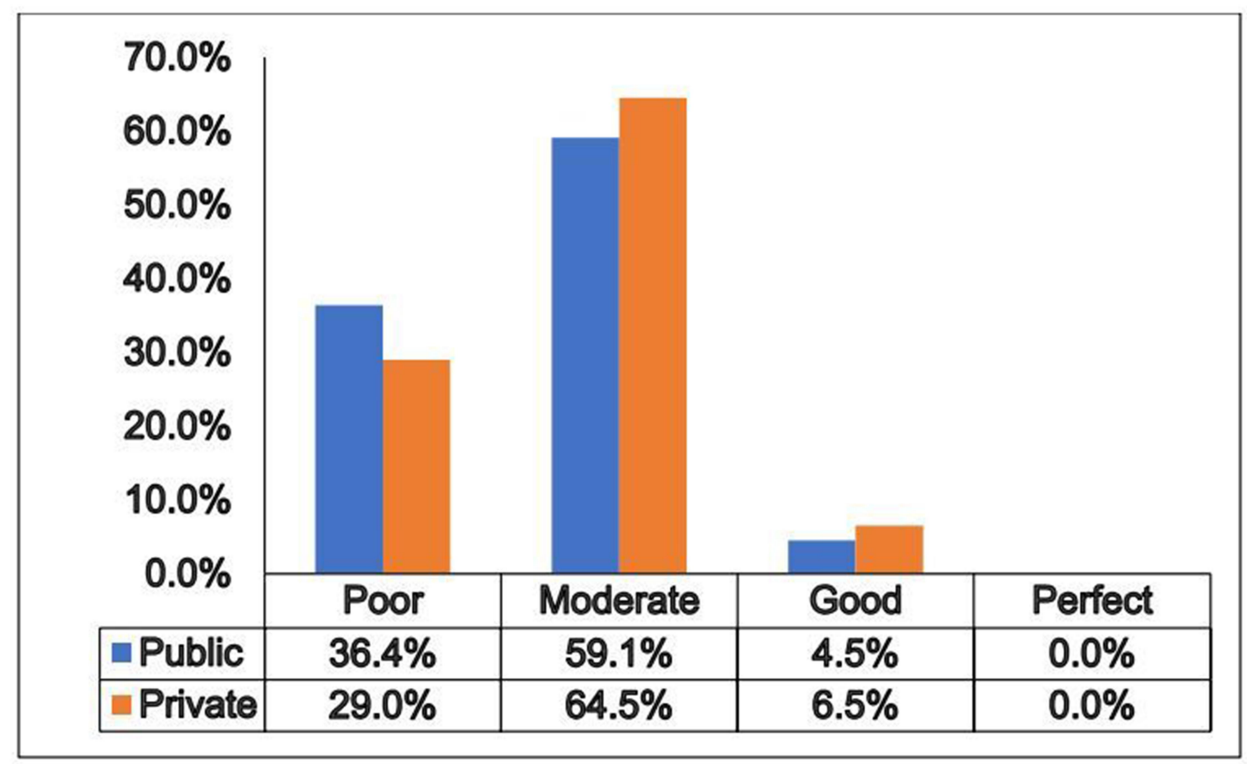

Figure I Percentage of public and private tertiary care hospitals in each defined category of adherence for facilities' indicators. 
Table I Core Elements of Outpatient Antibiotics Stewardship for Facilities

\begin{tabular}{|l|l|l|l|l|}
\hline \multirow{2}{*}{} & Mean (SD) & \multicolumn{3}{l|}{ Subgroup Analysis } \\
\cline { 3 - 5 } & & Public Hospitals Mean (SD) & Private Hospitals Mean (SD) & t* (p-value) \\
\hline Commitment & $1.58(1.00)$ & $1.50(0.96)$ & $1.65(1.05)$ & $-0.5 I(0.84)$ \\
Action & $1.42(1.39)$ & $1.4 I(1.14)$ & $1.42(1.57)$ & $-0.03(0.21)$ \\
Tracking and Reporting & $0.92(1.04)$ & $0.64(0.95)$ & $1.13(1.06)$ & $-1.77(0.05)$ \\
Education and Expertise & $1.66(1.12)$ & $1.4 I(I .01)$ & $1.84(1.19)$ & $-1.38(0.73)$ \\
Total score & $5.58(2.97)$ & $4.95(2.95)$ & $6.03(2.95)$ & $-1.31(0.37)$ \\
\hline Adherence Score Category & Moderate & Poor & Moderate & - \\
\hline
\end{tabular}

Notes: *Independent $t$-test. Bold values indicate statistical significance.

Abbreviation: SD, standard deviation.

subgroup analysis, clinician in public and private hospitals scored almost equal (public $=6.1$, private $=6.7, \mathrm{p}$-value $=$ 0.93). However, in terms of individual core elements, mean scores indicated that clinicians in public were more committed (commitment) for stewardship than clinicians in private hospitals. On the other hand, mean scores for private clinicians was higher in all other individual core elements, such as action, and tracking and reporting which recorded significant difference as detailed in Table 2 .

\section{Discussion}

\section{Summary}

The findings of this study clearly indicate that overall OPD facilities in Punjab has a poor to moderate adherence to CEOAS despite having a sizeable infrastructure, especially in the private sector. Out of four individual core elements which define facilities, major gaps were identified in tracking and reporting (the poorest mean score, i.e., 0.92, Table 1) of antibiotics prescribing, percentage of visits leading to antibiotic prescription, and antibiotic use and resistance trends in OPDs. Thus, tracking and reporting area emerges as a top priority area that requires immediate attention of the health authorities. Although the magnitude of the statistical significance for the tracking and reporting for facilities and for Action among the clinicians' indictors is at the borderline $(p=0.05)$, still depicts a trend towards significant difference between private and public hospitals and hence, must be given due consideration as a priority area. On the other hand, it is optimistic to learn that clinicians have mentioned good level of commitment and leadership for an improved stewardship in OPDs.

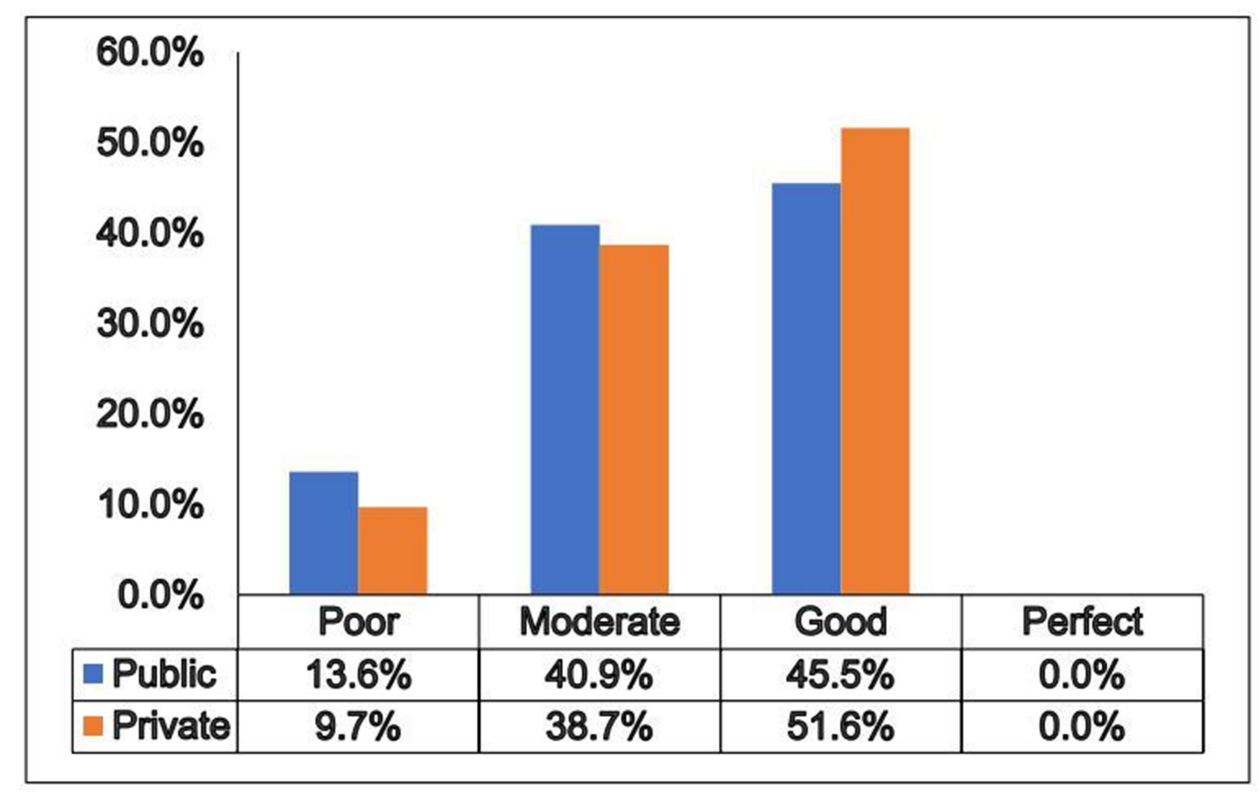

Figure 2 Percentage of public and private tertiary care hospitals in each defined category of adherence for clinicians' indicators. 
Table 2 Core Elements of Outpatient Antibiotics Stewardship for Clinicians

\begin{tabular}{|l|l|l|l|l|}
\hline \multirow{2}{*}{} & Mean (SD) & \multicolumn{3}{l|}{ Subgroup Analysis } \\
\cline { 3 - 5 } & & Public Hospitals Mean (SD) & Private Hospitals Mean (SD) & t* (p-value) \\
\hline Commitment & $1.17(0.99)$ & $1.27(0.98)$ & $1.10(1.01)$ & $0.63(0.22)$ \\
Action & $1.60(0.97)$ & $1.50(1.10)$ & $1.68(0.87)$ & $-0.63(\mathbf{0 . 0 5 )})$ \\
Tracking and Reporting & $1.64(0.94)$ & $1.45(1.06)$ & $1.77(0.84)$ & $-1.18(\mathbf{0 . 0 3 )}$ \\
Education and Expertise & $2.02(1.10)$ & $1.82(0.96)$ & $2.16(1.19)$ & $-1.12(0.37)$ \\
Total score & $6.43(2.61)$ & $6.05(2.63)$ & $6.71(2.61)$ & $-0.91(0.93)$ \\
\hline Adherence Score Category & Moderate & Moderate & Moderate & \\
\hline
\end{tabular}

Notes: *Independent $t$-test. Bold values indicate statistical significance.

Abbreviation: SD, standard deviation.

Nevertheless, top deficiency area identified in clinicians' indicator is education (mean score, 2.02, Table 2) that includes providing educational material to patients about antibiotic harms and misuse and continuous professional education on antibiotic prescribing for clinicians. These findings are correlated with the findings of another quantitative audit of the inappropriate prescribing of the antibiotics in hospitals in Lahore. ${ }^{17}$ Implementation of some form for ASPs in hospitals is an essential component of the One Health strategy to combat antibiotic resistance. However, results indicate that hospitals in Pakistan are facing complex challenges to implement ASPs which are not limited to resources only but also include, lack of expertise and human resource, complicated hierarchies within hospitals, and inability to track and report antibiotic consumption. ${ }^{5,17}$

After the 18th amendment in the constitution, Health is now a provincial responsibility and every province is autonomous to proffer and implement policies to improve the health care system. However, it is the Ministry of National Health Services, Regulation and Coordination (MoNHSR\&C) that defines standards and aims to meet international obligations. Pakistan has documented an appreciable response and developed its national action plan for antimicrobial resistance in 2017 that intends to serve as a blueprint for the activities, however, findings of this study imply that practically no steps have been taken out that could change significantly policy and practice in hospitals. Another factor which may influence is the seriousness' of political will, i.e., for decades, Pakistan has spent less than $1 \%$ of the GDP on health, which is one of the lowest in the region. ${ }^{15}$ In this regard, a heavy responsibility rests on the Antimicrobial Resistance Oversight Committee constituted by the MoNHSR\&C to provide funding to develop human resource, diagnostic capabilities and monitor the process of development and uptake of ASPs in hospitals at federal, provincial and district levels. Based on the best use of efforts and available resources, hospitals in Punjab ought to implement at least foundational yet sustainable ASPs with a vision to adhere all CEOAS gradually. The foundational core elements require only a dedicated focal person for coordination of stewardship activities and support from the hospital administration. An infectious disease physician or a clinical pharmacist may be an appropriate choice for the lead role of focal person. Having a senior physician advocating ASPs may be helpful in getting support of the higher hierarchy in the hospital administration. ${ }^{3,18}$ Furthermore, a multidisciplinary team of health care professionals may offer a skill mix required to achieve the objectives of stewardship activities. Nevertheless, a nurse, pharmacist or microbiologist trained in the area of infectious diseases could be a useful resource for ASPs in resource limited setting. ${ }^{19}$

\section{Comparison with the Existing Literature}

The findings of this study may be broadly comparable to some of the results reported in a recent study conducted in the hospitals of Punjab. ${ }^{20}$ Current findings are in agreement that major lacking exist in reporting and tracking of the antibiotics use. Authors do strongly advocate to adopt a standardized approach throughout Pakistan for the implementation of ASPs activities and for the same reason we evaluated the adherence on an international standard. Furthermore, education of masses and health care professionals are equally important. However, following differences and disagreements worth documenting: 1) The focus of the current study is specific to OPD in tertiary care hospitals only; 2) The response in this study is mainly taken from the physicians at administrative positions in the hospitals; 3) The difference in survey instrument is pertinent. The CDC proffered CEOAS has a standardized and comprehensive approach to evaluate the antimicrobial 
stewardship through two angles, i.e., institutional policies and commitment (facility indicator) and individual attitude and practices (physician indicators). Importantly, current findings contradict not only the assumption reported in the study cited above but also refute findings of another qualitative study in Punjab that highlightedlimited knowledge of ASPs among the physicians. ${ }^{11}$ The present study reports moderate to good level of adherence to CEOAS by the clinicians that inductively means they have good knowledge of the ASPs. Another possible explanation or reason of our findings could be the updated knowledge and momentum this issue (antibiotic resistance and ASPs) has gained in the health circles over a period of roughly a year (difference in the time period of data collection). Another point to establish here is, the individuals' knowledge may not play a significant role especially in the implementation of policies in accordance to the existing top-down organizational culture in majority of the institutions in Pakistan including hospitals. In-fact, the extent of implementation of AMSs programs in hospitals is largely determined by the national initiatives, the strength of commitment of policymakers and senior leadership in hospitals. ${ }^{21}$

At an international scale, a study conducted in the USA also aimed to evaluate the adherence of hospitals to CDC recommended new-born specific ASPs. ${ }^{22}$ However, authors noted one formidable difference in addition to the better adherence reported in the study and that was the use of auditors to conduct a cross-sectional audit which produced more reliable results. This is different from an academic investigation (as the case of this study) where the data collectors, contrary to auditors, have no authority to cross verify the facts stated in the survey. As a matter of fact, our study is an academic investigation and hence like any survey, possibilities of the socially desirable response bias in reporting of adherence could be there.

\section{Implications for Policy and Practice and Further Research}

Based on the findings, current study suggests following implications for policy, practice and further research:

(a) This study has highlighted certain deficiency areas in the practice of ASPs, such as tracking and reporting in facilities and education for clinicians and patients. These areas should be prioritized in any future policy shift and must be given due weightage. (b) MoNHSR\&C should take a step forward and enforce a minimum set standard of ASP, such as CEOAS, in OPDs of all tertiary care hospitals.

(c) Pakistan needs to deploy its huge underutilized health care workforce in the form of pharmacist and nurses in the antibiotic stewardship activities.

(d) Similar to this study, routine cross-sectional audits of hospitals are needed to keep a check on the progress of implementation of the ASPs.

\section{Limitations and Strength of the Study}

We noted the following limitations/strengths of the study.

(a) We only covered tertiary care hospitals in 8 administrative divisions of Punjab out of 9 due to short time period of an academic calendar. However, the missing division (Dera Ghazi Khan) was remotely located and health infrastructure or human resource (facilities or clinicians) could not significantly change the statistical results.

(b) The sample was though diverse and large (in context of the total number of tertiary care hospitals in Punjab) yet purposive, and the participation was voluntary and not an obligation as the case of government funded Audits, hence results may be interpreted with caution in terms of generalizability.

(c) We did not collect detailed demographic data of the hospitals, such as the number of beds, wards, lab facilities, etc. However, this kind of data were not much related to the objective set in this research and thus, may be of lesser value.

(d) Data were filled by the representatives of the administration of the hospitals. This might have introduced an element of slight exaggeration or socially desirable response bias.

\section{Strength}

(a) To our knowledge, it is the first study to report adherence of tertiary care hospitals to CEOAS in Punjab and Islamabad Capital Territory.

(b) Our study was free of recruitment bias as the data collectors did not opt for a convenient sample of the hospitals in Lahore only, rather reach out to hospitals diversely located in 8 administrative divisions of Punjab out of 9.

(c) Study reported specific gaps in ASPs in hospitals in Punjab for future policy. 
(d) Findings may be useful for other LMIC countries for an introspection point of view and urge health care stakeholders to encourage hospitals to implement a minimum set of ASPs.

\section{Conclusion}

There are significant gaps in the adherence to CEOAS in the tertiary care hospitals in Punjab, Pakistan. Overall, majority of the facilities in OPDs have poor to moderate, while, clinicians have moderate adherence to CEOAS. There are no significant differences between private and public hospitals for facilities and clinicians except in tracking and reporting for facilities and clinicians where private hospitals have slightly higher scores. The findings are though disappointing; however, hospitals and specially clinicians are getting more familiar about the need of ASPs which is demonstrated by the level of commitment in both private and public hospitals. The findings of this study have raised pertinent concerns for the relevant stakeholders. An immediate priority has emerged out of the finding of this study to foster and implement strategies to improve tracking and reporting of antibiotic use in hospitals. These gaps in ASPs highlighted in this study should be given substantial emphasis in future policy for hospital-based ASPs. Authors emphasize healthcare stakeholders in the country to take sizeable measures to improve and enforce implementation of ASPs in hospitals to implement at least foundational core elements which require no hefty resources. We can expect a significant impact only after majority of the OPDs in Pakistan adopt uniform policies and practices on ASPs under a common national framework. Implementation of ASPs may be challenging in the start, however, if left unattended, these gaps would lead to immense harm, and hence the time for action is now.

\section{Acknowledgment}

We extend our heartfelt thanks to Prof Dr Javaid Asgher, the CEO, Lahore Medical \& Dental College and Mr Asad Ahmad Khan, the Company Secretary, Lahore Medical \& Dental College for the valuable contacts to acquire data in various hospitals in the province.

\section{Author Contributions}

All authors made a significant contribution to the work reported, whether that is in the conception, study design, execution, acquisition of data, analysis and interpretation, or in all these areas; took part in drafting, revising or critically reviewing the article; gave final approval of the version to be published; have agreed on the journal to which the article has been submitted; and agree to be accountable for all aspects of the work.

\section{Funding}

This study received no financial support for the research, authorship, and/or publication of this article.

\section{Disclosure}

All authors declare no conflict of interest of any type for this work.

\section{References}

1. World Health Organization. WHO Report on Surveillance of Antibiotic Consumption: 2016-2018 Early Implementation. Geneva; 2018.

2. Roope LSJ, Smith RD, Pouwels KB, et al. The challenge of antimicrobial resistance: what economics can contribute. Science. 2019;364(6435):eaau4679. doi:10.1126/science.aau4679

3. Ostrowsky B, Banerjee R, Bonomo RA, et al. Infectious diseases physicians: leading the way in antimicrobial stewardship. Clin Infect Dis. 2018;66(7):995-1003. doi:10.1093/cid/cix1093

4. Fishman N. Policy statement on antimicrobial stewardship by the Society for Healthcare Epidemiology of America (SHEA), the Infectious Diseases Society of America (IDSA), and the Pediatric Infectious Diseases Society (PIDS). Infect Control Hosp Epidemiol. 2012;33(4):322-327. doi:10.1086/665010

5. Drekonja DM, Filice GA, Greer N, et al. Antimicrobial stewardship in outpatient settings: a systematic review. Infect Control Hosp Epidemiol. 2015;36(2):142-152. doi:10.1017/ice.2014.41

6. Public Health England. English Surveillance Programme for Antimicrobial Utilisation and Resistance (ESPAUR) 2018-2019; 2016:1-143

7. Arjun S. Antibiotic stewardship: why we must, how we can. Cleve Clin J Med. 2017;176(1):139-148. doi:10.1016/j.physbeh.2017. 03.040

8. Sanchez GV, Fleming-Dutra KE, Roberts RM, Hicks LA. Core elements of outpatient antibiotic stewardship. MMWR Recomm Rep. 2016;65(6):1-12. doi:10.15585/mmwr.rr6506a1

9. World Health Organization. Global Action Plan on Antimicrobial Resistance; 2015. doi:10.1128/microbe.10.354.1

10. Lee CF, Cowling BJ, Feng S, et al. Impact of antibiotic stewardship programmes in Asia: a systematic review and meta-analysis. $J$ Antimicrob Chemother. 2018;73(4):844-851. doi:10.1093/jac/dkx 492

11. Hayat K, Rosenthal M, Gillani AH, et al. Perspective of Pakistani physicians towards hospital antimicrobial stewardship programs: a multisite exploratory qualitative study. Int $J$ Environ Res Public Health. 2019;16(9):1-15. doi:10.3390/ijerph16091565

12. Hussain R, Hassali MA, Hashmi F, Farooqui M. A qualitative exploration of knowledge, attitudes and practices of hospital pharmacists towards adverse drug reaction reporting system in Lahore, Pakistan. J Pharm Policy Pract. 2018;11(1):1-10. doi:10.1186/ s40545-018-0143-0

13. Mubarak N. A multicentre point prevalence survey of the antibiotic use in tertiary care hospitals in Punjab; 2020:1-10.

14. Saleem Z, Hassali MA, Versporten A, et al. A multicenter point prevalence survey of antibiotic use in Punjab, Pakistan: findings and implications. Expert Rev Anti Infect Ther. 2019;1:1. doi:10.1080/14787210.2019.1581063

15. MoNHSRC. Antimicrobial Resistance. National Action Plan, Pakistan; 2017. 
16. Kamran R M, Syed SH, Babar ZUD, Ahmed SI. Extensively drug-resistant typhoid fever in pakistan macrolide resistance in streptococcus spp. Lancet Infect Dis. 2019;19(3):242-243. doi:10.1016/ S1473-3099(19)30051-9

17. Saleem Z, Saeed H, Hassali MA, et al. Pattern of inappropriate antibiotic use among hospitalized patients in Pakistan: a longitudinal surveillance and implications. Antimicrob Resist Infect Control. 2019;8(1):4-10. doi:10.1186/s13756-019-0649-5

18. Cox JA, Vlieghe E, Mendelson M, et al. Antibiotic stewardship in lowand middle-income countries: the same but different? Clin Microbiol Infect. 2017;23(11):812-818. doi:10.1016/j.cmi.2017.07.010

19. Barlam TF, Cosgrove SE, Abbo LM, et al. Implementing an antibiotic stewardship program: guidelines by the Infectious Diseases Society of America and the Society for Healthcare Epidemiology of America. Clin Infect Dis. 2016;62(10):e51-e77. doi:10.1093/cid/ ciw118
20. CDC. The core elements of human antibiotic stewardship programs in resource-limited settings: national and hospital levels; 2018.

21. Saleem Z, Hassali MA, Hashmi FK, Godman B, Ahmed Z. Snapshot of antimicrobial stewardship programs in the hospitals of Pakistan: findings and implications. Heliyon. 2019;5(7):e02159. doi:10.1016/j. heliyon.2019.e02159

22. Ho T, Buus-Frank ME, Edwards EM, et al. Adherence of newborn-specific antibiotic stewardship programs to CDC recommendations. Pediatrics. 2018;142(6):e20174322. doi:10.1542/ peds.2017-4322

\section{Publish your work in this journal}

Infection and Drug Resistance is an international, peer-reviewed openaccess journal that focuses on the optimal treatment of infection (bacterial, fungal and viral) and the development and institution of preventive strategies to minimize the development and spread of resistance. The journal is specifically concerned with the epidemiology of antibiotic resistance and the mechanisms of resistance development and diffusion in both hospitals and the community. The manuscript management system is completely online and includes a very quick and fair peerreview system, which is all easy to use. Visit http://www.dovepress.com/ testimonials.php to read real quotes from published authors. 